\title{
Structure of Polymeric Nanoparticles in Surfactant-Stabilized Aqueous Dispersions of High-Molar-Mass Hydrophobic Graft Polymers
}

Jana Hajduováa ${ }^{\mathrm{a}}$, Karel Procházka ${ }^{\mathrm{a}}$, Vladimír Raus ${ }^{\mathrm{b}}$, Miroslav Šlouf ${ }^{\mathrm{b}}$, Vladislav Krzyzanek ${ }^{\mathrm{c}}$, Vasil M. Garamus ${ }^{\mathrm{d}}$, and Miroslav Štěpánek ${ }^{\mathrm{a},{ }^{*}}$

${ }^{a}$ Department of Physical and Macromolecular Chemistry, Faculty of Science, Charles University in Prague, Hlavova 2030, 12840 Prague 2, Czech Republic

Fax: +420 22491 9752; Tel: +420 22195 1290; E-mail: stepanek@natur.cuni.cz

${ }^{b}$ Institute of Macromolecular Chemistry, Academy of Sciences of the Czech Republic,

Heyrovskýsq. 2, 16206 Prague 6, Czech Republic

${ }^{c}$ Institute of Scientific Instruments, Academy of Sciences of the Czech Republic,

Královopolská 147, 61264 Brno, Czech Republic

${ }^{d}$ Helmholtz-Zentrum Geesthacht, Centre for Materials and Coastal Research, D-21502

Geesthacht, Germany

\begin{abstract}
Aqueous dispersions of hydrophobic high-molar-mass cellulose-graft-polystyrene (Cel-g-PS) copolymers were prepared by nanoprecipitation from dioxane solutions using sodium dodecyl sulfate as a stabilizer. The size and structure of formed Cel-g-PS nanoparticles were studied by scattering techniques (static and dynamic light scattering, SAXS) and transmission electron microscopy. The scattering and microscopy data show that nanoprecipitation of Cel-g-PS leads to the formation of polydisperse aggregates of collapsed Cel-g-PS macromolecules containing entrapped SDS micelles in the kinetically frozen state. The final size of the aggregates can be tuned by changing the copolymer concentration, $c_{\mathrm{P}}$, in the dioxane solution. The average hydrodynamic radius of the aggregates, $R_{\mathrm{H}}$, scales as $\left[\left(R_{\mathrm{H}} / R_{\mathrm{H}, 0}\right)^{2.5}-1\right] \sim c_{\mathrm{P}}$, where $R_{\mathrm{H}, 0}$ is the $R_{\mathrm{H}}$ value extrapolated to the zero copolymer concentration.
\end{abstract}

\section{Introduction}

Polymer-surfactant interactions have been extensively studied in the last two decades with respect to the preparation of self-assembled nanoparticles and nanostructured materials with promising applications both in medicine and in technology. ${ }^{1-4}$ Many studies in this field have been focused to complexes of polyelectrolytes and oppositely charged ionic surfactants. Since polyelectrolyte-surfactant complexes are water-insoluble close to the zero net charge, 
co-assembly of block copolymers consisting of a polyelectrolyte block with oppositely charged surfactants allows for preparation of nanoparticles with the core of the insoluble complex and the shell of the neutral blocks. ${ }^{4-6}$

In the case of neutral hydrophobic polymers, surfactants are often used as stabilizers in order to prepare polymeric nanoparticles in aqueous media. These can either be prepared by emulsion polymerization, in which nanoparticles are formed from hydrophobic monomer nanodroplets dispersed in the aqueous medium, ${ }^{7-9}$ or by nanoprecipitation, which consists in formation of polymer nanoparticles by microphase separation of the polymer after mixing the polymer solution in a water-miscible organic solvent with water. The latter technique, which has been widely used in pharmacology for preparation of carrier vessels for drug delivery, ${ }^{10-12}$ is the subject of this paper.

Even though there are a number of articles about nanoprecipitation of polymers, most of them aim at encapsulation properties and pharmacological applications, while only a very few of them are focused on the structure of nanoprecipitated particles. ${ }^{13}$ Furthermore, nanoprecipitation studies usually deal with low-molar-mass polymers which behave as liquids and coalesce into compact particles, ${ }^{14}$ the size of which scales with the aggregation number as $R \sim N_{\mathrm{agg}}^{1 / 3}$. In this paper, we present a study of nanoparticles prepared by nanoprecipitation of cellulose-graft-polystyrene (Cel-g-PS) copolymers with the molar mass of the order of $10^{6} \mathrm{~g} \mathrm{~mol}^{-1}$ and high grafting densities. Our goal is to address the question whether the nanoprecipitation of such large dense macromolecules occurs as coalescence or whether it rather resembles the aggregation of solid particles. In the latter case, the aggregation process results in formation of fractal particles, so that $R \sim N_{\mathrm{agg}}{ }^{1 / d}$, where $d$ is the fractal dimension which is about 1.8 for diffusion-limited aggregation process. ${ }^{15}$

We use two Cel-g-PS samples differing in the grafting density and sodium dodecyl sulfate (SDS) as a stabilizing surfactant. The structure of the nanoparticles is investigated by microscopic (TEM) and scattering (light scattering, SAXS) techniques. We also study the dependence of the size of the particles on the concentration of the copolymer in dioxane solution and the surfactant in the aqueous solution.

\section{Experimental Part}

Materials. Polymer Synthesis. Cellulose-graft-polystyrene (Cel-g-PS) copolymers (Scheme 1) were prepared by the "grafting-from" technique by atom transfer radical 
polymerization using the macroinitiator prepared by acylation of microcrystalline cellulose by 2-bromoisobutyryl bromide under homogeneous reaction conditions in the $\mathrm{N}, \mathrm{N}$ dimethylacetamide/LiCl solvent system. Two samples differing in the grafting density were used for the study (Table 1). Details on the synthesis and characterization of the samples are given in ref. 16.

\section{Scheme 1. Structure of Cellulose-graft-Polystyrene Copolymers}

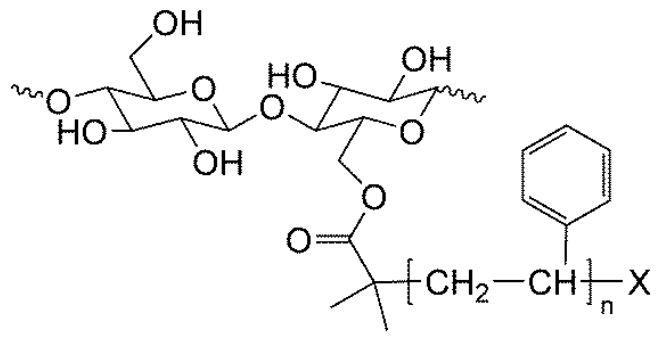

Preparation of Cel-g-PS Aqueous Dispersions. Cel-g-PS aqueous dispersions were prepared by adding $0.1 \mathrm{~mL}$ of the Cel-g-PS solution $(1-10 \mathrm{mg} / \mathrm{mL})$ in 1,4-dioxane dropwise into $0.9 \mathrm{~mL}$ of aqueous sodium dodecyl sulfate (SDS) solution $(1-100 \mathrm{mM})$ under vigorous stirring. Excess SDS was removed by dialysis against deionized water. The $\zeta$-potential of the nanoparticles in aqueous dispersions was found to be about $-30 \mathrm{mV}$ and independent of the SDS concentration in the aqueous solution before the dialysis. The dispersions prepared from $10 \mathrm{mg} / \mathrm{mL}$ Cel-g-PS dioxane solutions and $0.1 \mathrm{M}$ aqueous SDS (the final concentration of the copolymers in the dispersion was thus $1 \mathrm{mg} / \mathrm{mL}$ ) were used for electron microscopy and SAXS measurements. For LS measurements, the dispersions were further diluted with deionized water to achieve the final copolymer concentration $0.1 \mathrm{mg} / \mathrm{mL}$.

Table 1. Characterization of Cel-g-PS-I and Cel-g-PS-II copolymers

\begin{tabular}{|l|l|l|l|l|l|}
\hline Sample & $M_{\mathrm{n}}^{\text {gr }}, \mathrm{kg} \mathrm{mol}^{-1 \mathrm{a}}$ & $M_{\mathrm{w}} / M_{\mathrm{n}}^{\mathrm{b}}$ & $g^{\mathrm{c}}$ & $M_{\mathrm{n}}^{\text {th }}, 10^{3} \mathrm{~kg} \mathrm{~mol}^{-1 \mathrm{~d}}$ & $M_{\mathrm{w}}^{\text {th }}, 10^{3} \mathrm{~kg} \mathrm{~mol}^{-1 \mathrm{e}}$ \\
\hline Cel-g-PS-I & 12.2 & 1.35 & 0.42 & 0.587 & 1.15 \\
\hline Cel-g-PS-II & 11.2 & 1.18 & 1.04 & 1.182 & 2.32 \\
\hline
\end{tabular}

${ }^{a}$ number averaged molar mass of a PS graft by SEC, bdispersity index of a PS graft by SEC, 'grafting density, ${ }^{\mathrm{d}}$ theoretical number-averaged molar mass of the copolymers, $M_{\mathrm{n}}^{\text {th }}=\left(M_{\mathrm{b}} / M_{\mathrm{gl}}\right) g\left(M_{\mathrm{n}}^{\text {graft }}+M_{\mathrm{gl}}\right)$, where $M_{\mathrm{b}}=16.2 \mathrm{~kg}$ $\mathrm{mol}^{-1}$ is the number-averaged molar mass of the backbone and $M_{\mathrm{gl}}=0.162 \mathrm{~kg} \mathrm{~mol}^{-1}$ is the molar mass of the glucose unit, ${ }^{d}$ theoretical number-averaged molar mass of the copolymers assuming the dispersity index $\oslash=$ 1.96 calculated from the dispersity indices of the backbone and the grafts. ${ }^{17}$ 
Methods. Light Scattering (LS). The light scattering setup (ALV, Langen, Germany) consisted of a $22 \mathrm{~mW} \mathrm{He-Ne} \mathrm{laser,} \mathrm{operating} \mathrm{at} \mathrm{the} \mathrm{wavelength} \lambda=632.8 \mathrm{~nm}$, an ALV CGS/8F goniometer, an ALV High QE APD detector and an ALV 5000/EPP multibit, multitau autocorrelator. Both static and dynamic LS measurements were carried out at $25^{\circ} \mathrm{C}$ in the angular range $30^{\circ}$ to $150^{\circ}$ corresponding in aqueous solutions to the scattering vector magnitudes $q$ ranging from $6.8 \mu \mathrm{m}^{-1}$ to $25.6 \mu \mathrm{m}^{-1}$.

Refractive index increment measurements were carried out at $25^{\circ} \mathrm{C}$ using a Wyatt $\mathrm{T}$ ReX differential refractometer. The aqueous solutions of FSN-100 in four different concentrations were pumped into the refractometer by a syringe driven by a 78-9100C Cole Parmer syringe drive with a flow rate of $1 \mathrm{~mL} / \mathrm{min}$. The refractive index value, $\mathrm{d} n / \mathrm{d} c=0.17$ $\mathrm{ml} \mathrm{g}^{-1}$ was determined from the slope of the plot of the refractive index versus concentration.

Static light scattering (SLS) measurements of Cel-g-PS solutions in dioxane were treated by the Berry method using the equation

$$
\left[\frac{K c}{\Delta R_{\theta}(q, c)}\right]^{1 / 2}=\frac{1}{M_{\mathrm{w}}^{1 / 2}}\left(1+\frac{1}{6} R_{\mathrm{g}}^{2} q^{2}+\ldots\right)^{1 / 2}\left(1+M_{\mathrm{w}} A_{2} c\right)
$$

where $M_{\mathrm{w}}$, and $A_{2}$, respectively, are the weight-averaged molar mass, $R_{\mathrm{g}}{ }^{2}$ is the $z$-averaged squared radius of gyration and $A_{2}$ is the "light-scattering-averaged" osmotic second virial coefficient of the polymer in the solution, $\Delta R \theta(q, c)$ is the corrected Rayleigh ratio which depends on the polymer concentration $c$ and on the magnitude of the scattering vector $q=\left(4 \pi n_{0} / \lambda\right) \sin (\theta / 2)$, where $\theta$ is the scattering angle, $n_{0}$ is the refractive index of the solvent and $\lambda$ is the wavelength of the incident light. The contrast factor $K$ is given by the relationship $K=4 \pi^{2} n_{0}^{2}(\mathrm{~d} n / \mathrm{d} c)^{2} /\left(\lambda^{4} N_{\mathrm{A}}\right)$, where $\mathrm{d} n / \mathrm{d} c$ is the refractive index increment of the polymer with respect to the solvent, and $N_{\mathrm{A}}$ is the Avogadro constant.

Dynamic light scattering measurements were evaluated by fitting the measured normalized time autocorrelation function of the scattered light intensity, $g^{(2)}(t)$, related to the electric field autocorrelation function, $g^{(1)}(t)$, by the Siegert relation, $g^{(2)}(t)=1+\beta\left|g^{(1)}(t)\right|^{2}$.

The data, collected for various copolymer concentrations and scattering angles were fitted (i) with the aid of the constrained regularization algorithm (CONTIN) which provides the distribution of relaxation times $\tau, A(\tau)$, as the inverse Laplace transform of $g^{(1)}(t)$ function 


$$
g^{(1)}(t)=\int_{0}^{\infty} A(\tau) \exp \left(-\frac{t}{\tau}\right) \mathrm{d} \tau
$$

and (ii) to the second order cumulant expansion

$$
g^{(1)}(t)=\exp \left(-\Gamma_{1} t+\frac{\Gamma_{2}}{2} t^{2}\right)
$$

where $\Gamma_{1}$ and $\Gamma_{2}$, respectively, are the first and the second moment of the distribution function of relaxation rates. The z-averaged diffusion coefficient of the particles, $\langle D\rangle_{z}$, was obtained by the linear extrapolation to zero $q$ and $c$ values as

$$
\frac{\Gamma_{1}(q, c)}{q^{2}}=D_{z}\left(1+C R_{\mathrm{g}}^{2} q^{2}+\ldots\right)\left(1+k_{\mathrm{D}} c\right)
$$

where $C$ is the structure parameter dependent of the shape and degree of polydispersity of the particles and $k_{\mathrm{D}}$ is the hydrodynamic virial coefficient.

The average hydrodynamic radius (the z-average of $R_{\mathrm{H}}{ }^{-1}$ ) was calculated from $D_{\mathrm{z}}$, by means of the Stokes-Einstein formula

$$
R_{\mathrm{H}}=\frac{k_{\mathrm{B}} T}{6 \pi \eta_{0} D_{\mathrm{z}}},
$$

where $k_{\mathrm{B}}$ is the Boltzmann constant, $T$ is the temperature and $\eta_{0}$ is the viscosity of the solvent. The $A(\tau)$ distributions can be recalculated to the distributions of apparent hydrodynamic radii, $R_{\mathrm{H}}{ }^{\text {app }}$, using the relationship

$$
R_{\mathrm{H}}^{\mathrm{app}}=\frac{k_{\mathrm{B}} T q^{2}}{6 \pi \eta_{0}} \tau
$$

Electrophoretic Light Scattering. $\zeta$-Potential measurements were carried out with a Nano-ZS Zetasizer (Malvern Instruments, U.K.). $\zeta$-Potential values were calculated from electrophoretic mobilities (average of three subsequent measurements, each of which 
consisted of 15-100 runs) using the Henry equation in the Smoluchowski approximation, $\mu=$ $\varepsilon \zeta / \eta$, where $\mu$ is the electrophoretic mobility and $\varepsilon$ is the dielectric constant of the solvent.

Transmission Electron Microscopy (TEM). TEM micrographs were obtained with a Tecnai G2 Spirit Twin 12 microscope (FEI, Czech Republic) at $120 \mathrm{kV}$ using bright field imaging and various tilt angles $\left(0^{\circ}\right.$ and $\left.50^{\circ}\right)$. The samples were prepared as follows: $2 \mu \mathrm{L}$ of Cel-g-PS copolymer solution in dioxane or nanoparticle dispersion in water (the copolymer concentration was, $c_{\mathrm{P}}=1 \mathrm{mg} / \mathrm{mL}$, in both cases) were dropped onto a copper TEM grid (300 mesh) coated with thin, electron-transparent carbon film. The solution was sucked out by touching the bottom of the grid with filtering paper. This fast removal of the solution was performed after $1 \mathrm{~min}$ in order to suppress oversaturation during the drying process.

Small-angle X-Ray Scattering (SAXS). SAXS experiments were performed on the P12 BioSAXS beamline at the storage ring PETRA III of the Deutsche Elektronen Synchrotron (DESY, Hamburg, Germany) at $20^{\circ} \mathrm{C}$ using a Pilatus $2 \mathrm{M}$ detector and synchrotron radiation with the wavelength of $\lambda=0.12 \mathrm{~nm}$. The sample-detector distance was $4.1 \mathrm{~m}$, allowing for measurements in the $q$-range interval from 0.07 to $4.6 \mathrm{~nm}^{-1}$. The $q$ range was calibrated using the diffraction patterns of silver behenate. A sample of approximately $20 \mu \mathrm{l}$ was illuminated. The experimental data were normalized to the incident beam intensity, corrected for nonhomogeneous detector response, and the background scattering of the solvent was subtracted. The solvent scattering was measured before and after the sample scattering to control for possible sample holder contamination. We recorded 20 diffraction patterns originating from the same sample volume, using an exposure time of $0.05 \mathrm{~s}$. This backgroundcorrected SAXS data were used to calculate one-dimensional scattering curves by angular averaging.This background-corrected SAXS data were used to calculate one-dimensional scattering curves by angular averaging using an automated acquisition and analysis program [ Franke D, Kikhney AG, Svergun DI (2012) Automated acquisition and analysis of small angle X-ray scattering data. Nucl Instrum Methods A 689: 52-59.]. 


\section{Results and Discussion}

Characterization of Cel-g-PS copolymers by light scattering. Prior to the preparation of nanoprecipitated particles, both Cel-g-PS copolymers were studied in 1,4-dioxane solutions at concentrations ranging from 1 to $10 \mathrm{mg} / \mathrm{mL}$ by static and dynamic $\mathrm{LS}$ in order to determine their molar mass, size and interaction parameters. Berry plots and dynamic Zimm plots of the scattering data for both copolymers are shown in Fig. 1. They are qualitatively similar for both samples with the exception of the sign of the hydrodynamic virial coefficient, which indicates that in the case of Cel-g-PS-I, the thermodynamic contribution to $k_{\mathrm{D}}$ is lower than the friction contribution, oppositely to the case of Cel-g-PS-II ( $k_{\mathrm{D}}=M_{\mathrm{w}} A_{2}-k_{\mathrm{f}}-v$, where $k_{\mathrm{f}}$ is the friction coefficient and $v$ is the molar volume of the scattering particles). Results obtained from the evaluation of the scattering data (eqs. 1,4,5) are summarized in Table 2.

Table 2. Characteristics of Cel-g-PS Copolymers Obtained by LS Measurements in 1,4-Dioxane Solutions

\begin{tabular}{|l|l|l|l|l|l|}
\hline sample & $M_{\mathrm{w}}, 10^{6} \mathrm{~g} \mathrm{~mol}^{-1}$ & $R_{\mathrm{g}}, \mathrm{nm}$ & $A_{2}, 10^{-9} \mathrm{~mol} \mathrm{dm}^{3} \mathrm{~g}^{-2}$ & $R_{\mathrm{H}}, \mathrm{nm}$ & $k_{\mathrm{D}}, 10^{-2} \mathrm{dm}^{3} \mathrm{~g}^{-1}$ \\
\hline Cel-g-PS-I & 4.7 & 79 & 9.78 & 56 & -2.83 \\
\hline Cel-g-PS-II & 20.0 & 113 & 6.74 & 83 & 3.61 \\
\hline
\end{tabular}
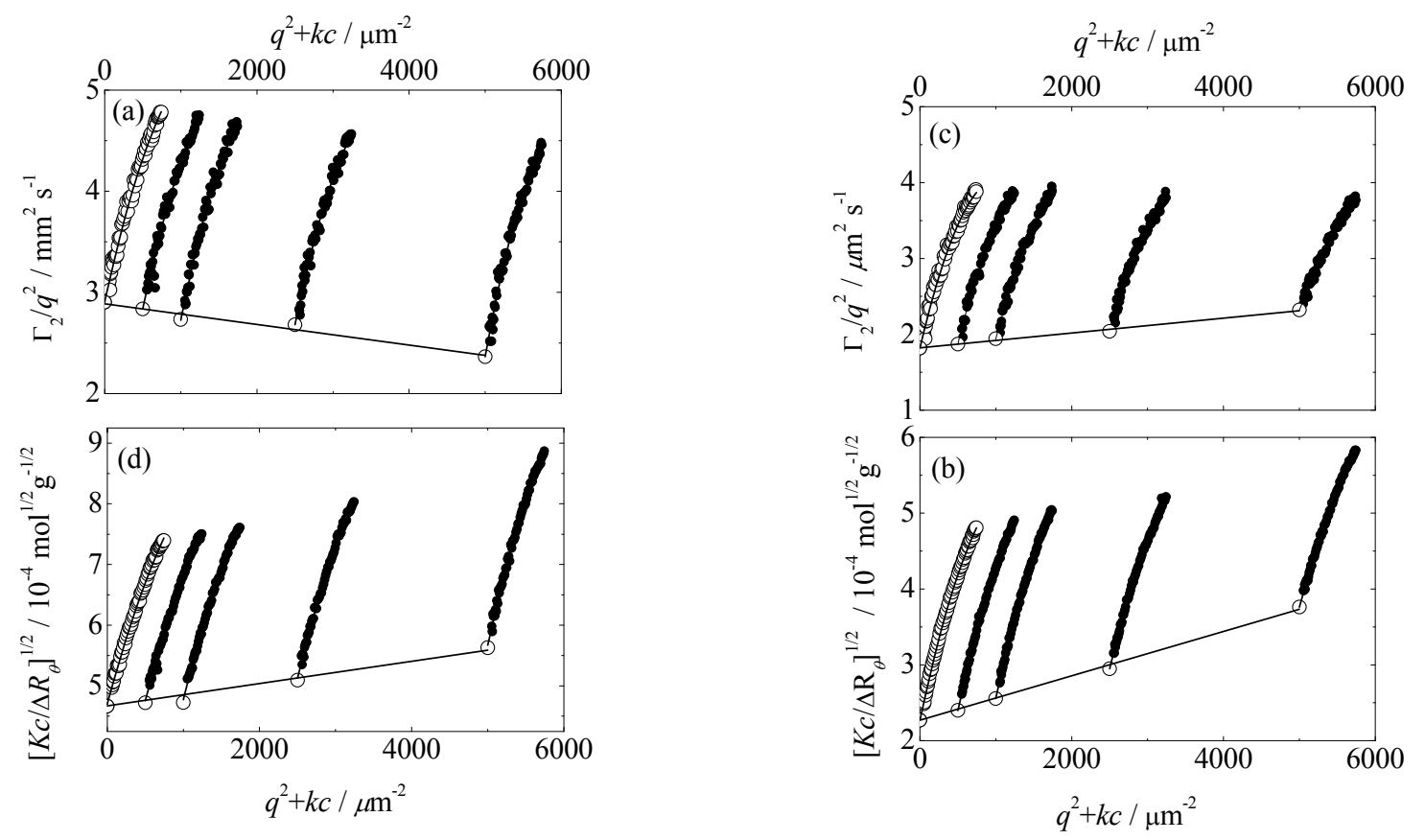
Fig. 1. Dynamic Zimm plots $(a, c)$ and Berry plots $(b, d)$ of the static and dynamic light scattering data from solutions of Cel-g-PS-I (a,b) and Cel-g-PS-II (c,d) in 1,4-dioxane.

Similarly to the previous study, ${ }^{16}$ the molar masses of both samples are significantly higher than those calculated from the known grafting densities and molar masses of the grafts and backbones. Moreover, the molar mass of Cel-g-PS-II is more than four times higher than that of Cel-g-PS-I, although according to the grafting densities, $M_{\mathrm{w}}$ of both samples should differ only by the factor of approximately two. It has been hypothesized that the discrepancy between calculated $M_{\mathrm{w}}^{\text {theor }}$ and measured $M_{\mathrm{w}}$ can be ascribed to a partial intermolecular recombination of growing polystyrene chains, resulting in covalent bonding between different copolymer molecules. ${ }^{16}$ Since the probability of the formation of a crosslink of two backbones via the recombination of the grafts increases with the increasing number of grafts, this assumption is supported by the fact that the difference between $M_{\mathrm{w}}{ }^{\text {theor }}$ and measured $M_{\mathrm{w}}$ is larger for the sample with the higher grafting density.

Cel-g-PS Nanoparticles in Aqueous Dispersions. Light and small-angle X-ray scattering were used in order to elucidate the structure of Cel-g-PS nanoparticles in water. Fig. 2 shows LS curves of aqueous dispersions of Cel-g-PS-I and Cel-g-PS-II. In the $q$ range covered by the LS measurements, corresponding to the lengthscales from ca. 800 to ca. 250 $\mathrm{nm}$, an intermediate regime between the Guinier and power-law scattering behavior is observed. Fitting the curves by the Fisher-Burford structure factor, ${ }^{20}$

$$
I(q)=I_{0}\left(1-\frac{2}{3 \alpha_{1}} R_{\mathrm{g}}^{2} q^{2}\right)^{\alpha_{1} / 2}
$$

where $I_{0}$ is the forward scattering intensity, $R_{\mathrm{g}}$ is the gyration radius and $\alpha_{1}$ is the exponent in the power law regime, provides the values of the particle gyration radius, $260 \mathrm{~nm}$ for Cel-gPS-I and $269 \mathrm{~nm}$ for Cel-g-PS-II. The gyration radii can be compared with the results of dynamic LS measurements (Fig. 3). The obtained apparent diffusion coefficients are strongly $q$-dependent. While the increase of $D_{\text {app }}$ with $q$ is due to polydispersity of the scatterers, the decreasing part of the dependence for high $q$ is caused by back reflected light. ${ }^{21}$ Hydrodynamic radii calculated from the diffusion coefficients extrapolated to zero $q$ are 226 $\mathrm{nm}$ for Cel-g-PS-I and $238 \mathrm{~nm}$ for Cel-g-PS-II, yielding the $R_{\mathrm{g}} / R_{\mathrm{H}}$ ratios, $\rho, 1.15$ for Cel-gPS-I and 1.13 for Cel-g-PS-II. 


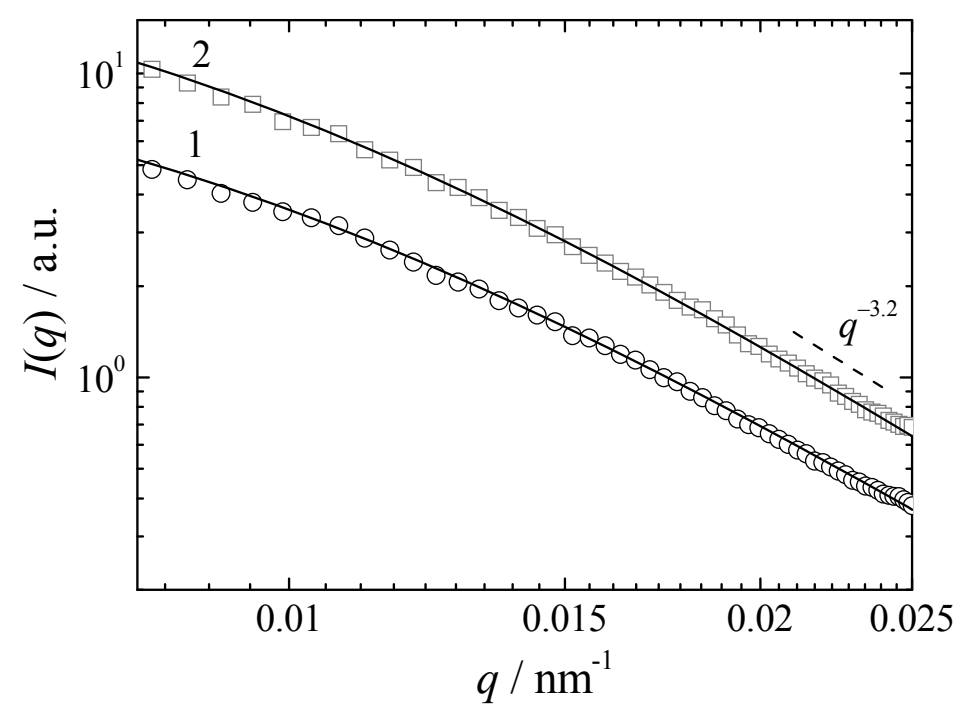

Fig. 2. Static LS curves for Cel-g-PS-I (curve 1) and Cel-g-PS-II (curve 2) aqueous dispersions.

The power-law exponent $\alpha_{1}$ obtained from the fits of the eq. 7 is 3.25 for Cel-g-PS-I and 3.46 for Cel-g-PS-II. Since $-3>\alpha_{1}>-4$, the scattering above the Guinier region comes from the surface of the aggregates. The values of $\alpha_{1}$ lower than 4 generally indicate the rough surface (with the surface fractal dimension $d_{\mathrm{s}}=6+\alpha_{1}$ ) but the decreased power-law exponent can also be a result of the polydispersity of the system.

In the case of Cel-g-PS dispersions, the surface fractal dimension of the aggregates can be influenced by both factors. Firstly, the large collapsed Cel-g-PS macromolecules are not flexible and cannot merge into an aggregate with the sharp interface. Secondly, since the average $R_{\mathrm{g}}$ of the aggregates is only 3-5 times larger than that of the Cel-g-PS macromolecules in the dioxane solutions, the aggregation numbers are low and fluctuations in $N_{\text {agg }}$ will result in the broad size distribution of the aggregates. Moreover, also the individual Cel-g-PS macromolecules are polydisperse due to the above-mentioned formation of crosslinks between the cellulose backbones.

Fig. 4 shows SAXS scattering curves in the $q$ range corresponding to the lengthscale from 104 to $2 \mathrm{~nm}$ which probe the internal structure of the collapsed particles forming the aggregate. The scattering in this region exhibits the power law behavior, except for the high- $q$ region where a weak peak appears for both dispersions. Treating the correlation peak as the simple Lorentzian function (the structure factor for disordered cell-cell correlations ${ }^{22}$ ), the curve can be fitted by the following empirical scattering function 


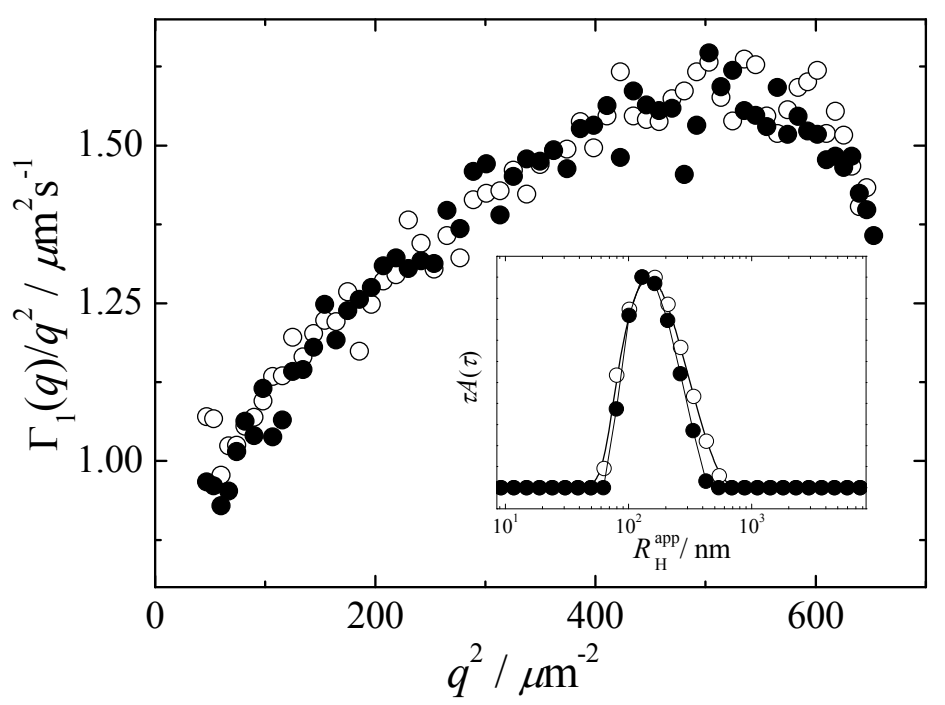

Fig. 3. Dynamic Zimm plots for Cel-g-PS-I (solid circles) and Cel-g-PS-II (open circles) in aqueous dispersions. Insert: CONTIN distributions of apparent hydrodynamic radii for Cel-g-PS-I (solid circles) and Cel-g-PS-II (open circles) at the scattering angle $\theta=90^{\circ}$.

$I(q)=I_{0} q^{\alpha_{2}}+\frac{I_{1} \xi^{-2}}{\xi^{-2}+\left(q-q_{\mathrm{c}}\right)^{2}}$,

where $I_{0}$ and $I_{1}$ are prefactors, $\xi$ is the full width in the half-maximum of the peak (the correlation length) and $q_{\mathrm{c}}$ is the center of the peak. The fit provides the values of the exponent $\alpha_{2}$ about -2.6 ( -2.53 for Cel-g-PS-I and -2.63 for Cel-g-PS-II), indicating that the particles have a dense, but not fully collapsed structure with the mass fractal dimension $d_{\mathrm{m}}=-\alpha_{2}$.

The maxima of the peaks, $q_{\mathrm{c}}$, are $1.44 \mathrm{~nm}^{-1}$ for Cel-g-PS-I and $1.67 \mathrm{~nm}^{-1}$ for Cel-gPS-II and indicate a local ordering at the lengthscale, $l=2 \pi / q_{\mathrm{c}}$, of ca. 3-4 nm. Since this value is close to the diameter of SDS micelles, the peak suggests the presence of the packed SDS micelles in the particles (similarly to polyelectrolyte-surfactant complexes, in which this behavior can be much more pronounced ${ }^{23}$ ). Let us remind that the dispersions remain stable (the particles keep the negative zeta potential of about $-30 \mathrm{mV}$ ) even though they undergo dialysis during which free surfactant is removed from the solution. This behavior clearly indicates that the Cel-g-PS/SDS complex is in a kinetically frozen state so that a fraction of SDS remains trapped in the interior of the collapsed Cel-g-PS macromolecules. 


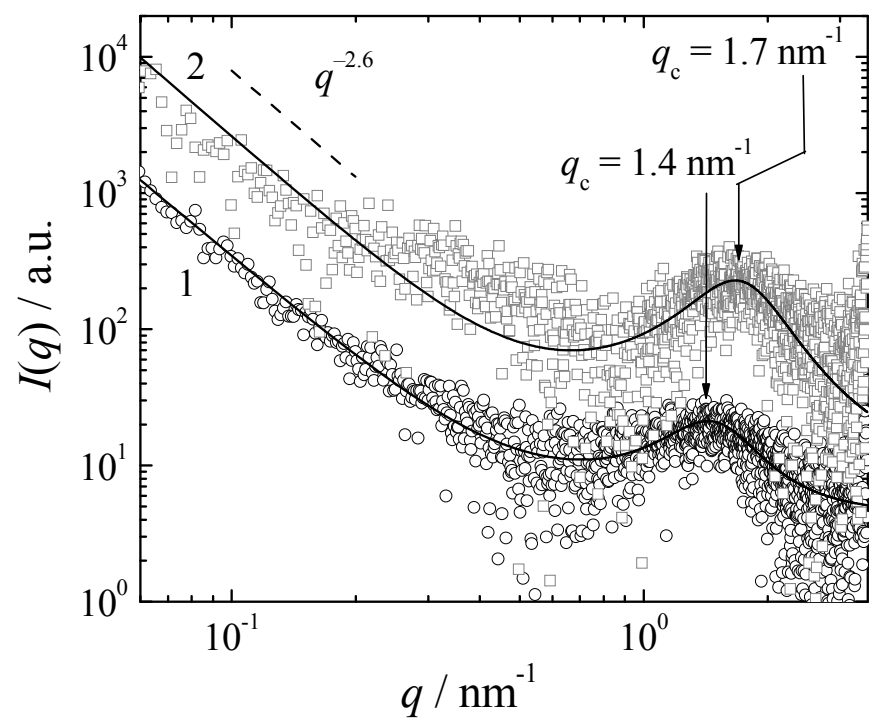

Fig. 4. SAXS curves for Cel-g-PS-I (curve 1) and Cel-g-PS-II (curve 2) aqueous dispersions.

Transmission Electron Microscopy. TEM was used to visualize the structure of Cel-gPS macromolecules before (Fig. 4a-d) and after (Fig. 5a-d) the nanoprecipitation process. The aggregation was observed not only on the micrographs of the samples obtained by drying of the aqueous dispersions (Fig. 5a-d) but also on those prepared from the solutions in 1,4dioxane (Fig. 4a-d) which suggests that the observed aggregates form rather as a result of the deposition of the copolymer on the TEM grid and drying.

The micrographs clearly show that the mean diameter of the particles from the aqueous dispersions (about $170 \pm 80 \mathrm{~nm}$ for both Cel-g-PS-I and Cel-g-PS-II) is smaller than those from 1,4-dioxane (about $100 \pm 40 \mathrm{~nm}$ ). This difference suggests that the large particles deposited from the 1,4-dioxane solutions are fused aggregates of several Cel-g-PS macromolecules. In the case of aqueous dispersions, the fusion is not possible because the collapsed macromolecules loaded with the surfactant lack flexibility to do so. This explanation is supported by the micrographs obtained at the tilt angle of $50^{\circ}$, which reveal that the nanoprecipitated macromolecules from the aqueous dispersions are less flattened than those deposited from the dioxane solutions. 


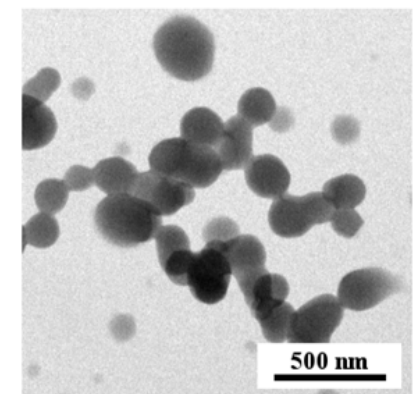

(a)

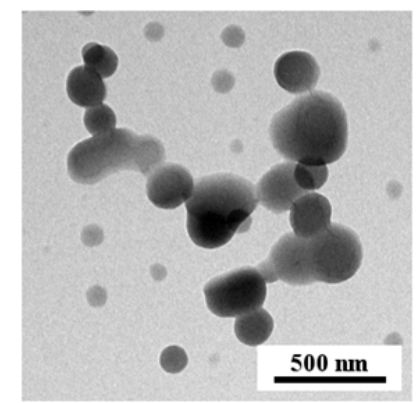

(c)

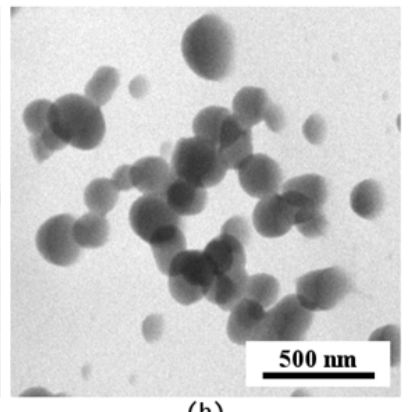

(b)

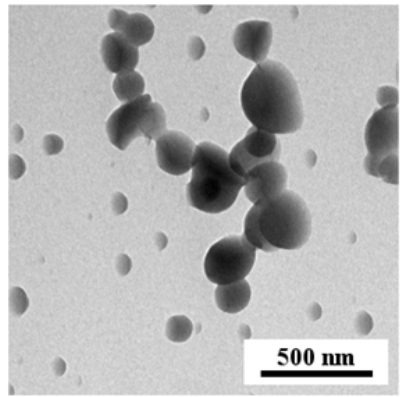

(d)

Fig. 4. Transmission electron micrographs of Cel-g-PS macromolecules from 1,4-dioxane solutions: (a,b) Cel-gPS-I, (c,d) Cel-g-PS-II, at tilt angles of $0^{\circ}(\mathrm{a}, \mathrm{c})$ and $50^{\circ}(\mathrm{b}, \mathrm{d})$.

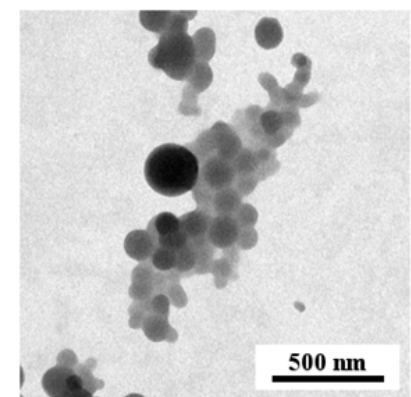

(a)

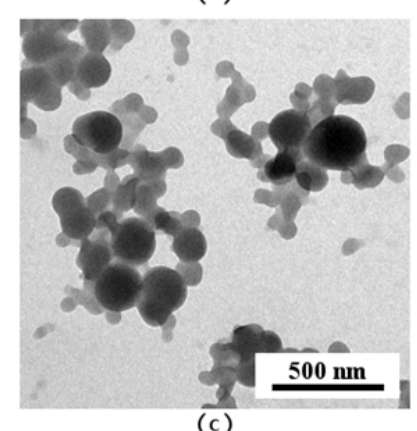

(c)

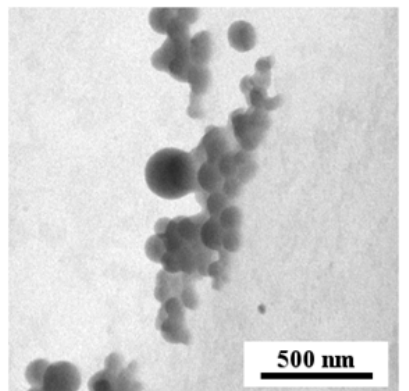

(b)

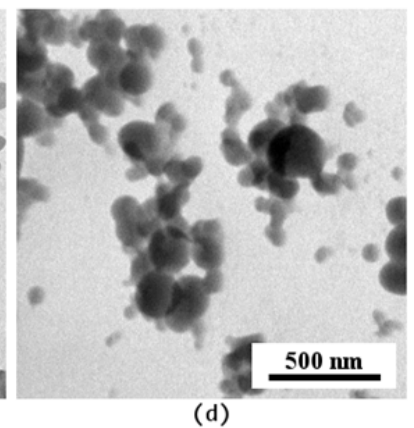

Fig. 5. Transmission electron micrographs of Cel-g-PS nanoparticles from aqueous dispersions: (a,b) Cel-g-PSI, (c,d) Cel-g-PS-II, at tilt angles if $0^{\circ}(\mathrm{a}, \mathrm{c})$ and $50^{\circ}(\mathrm{b}, \mathrm{d})$.

In order to study the influence of the sample preparation process on the observed particles, we employed another two scanning transmission electron microscopy (STEM) techniques: (i) In the case of 1,4-dioxane solutions, the samples were freeze dried and observed in high vacuum STEM. (ii) In the case of the aqueous dispersions, we studied the hydrated state by means of wet-STEM (STEM in the environmental mode as described in 
Supporting information). In both cases, the obtained images corresponded to those obtained by fast-drying at the room temperature followed by high-vacuum TEM microscopy. For the results and the experimental details of STEM techniques, see the Supporting Information.

Dependence of Cel-g-PS Particle Size on Copolymer and Surfactant Concentration. Since the formation of the nanoprecipitated particles is controlled both by the aggregation of Cel-g-PS macromolecules and by the adsorption of SDS on the formed aggregates which hinders the aggregation process, it can be expected that the size of the resulting particles will change with changing the initial polymer and SDS concentrations, $c_{\mathrm{P}}$ (from 1 to $10 \mathrm{mg} / \mathrm{mL}$ ) and $\underline{c}_{\mathrm{SDS}}$ (from 1 to $100 \mathrm{mM}$ ), in the preparation protocol described in the Experimental Section. Let us first consider the case of the size dependence on $c_{\mathrm{P}}$ in the excess of the surfactant. In this case, long-range electrostatic interactions are suppressed due to the high ionic strength of the solution and the aggregation process is then limited only by the diffusion of the copolymer macromolecules. (This does not contradict the observed fractal dimension of the aggregates which is higher than that corresponding to the diffusion-limited cluster aggregation regime ${ }^{15}$ because in our case the fractal dimension is determined mainly by the internal structure of the collapsed Cel-g-PS macromolecules and polydispersity of the aggregates.) For the diffusion-limited coalescence process, ${ }^{14}$ the characteristic size of the aggregates grows with the time as $R^{3}(t)=R_{0}{ }^{3}[1+t / \tau]$, where $\tau$ is the characteristic coalescence time which is inversely proportional to the concentration of the copolymer, $c_{\mathrm{P}}$, and $R_{0}$ is the initial size of the particles, that is, the size of the singe collapsed Cel-g-PS macromolecule. Therefore, taking into account that in our case the aggregation number scales with the size as $N_{\text {agg }} \sim R^{d}$, where $d=2.53$ is the mass fractal dimension, and assuming further that the characteristic time of mixing at which the aggregates reach their maximum size is independent on $c_{\mathrm{P}}$, the increase in the particle size with the copolymer concentration should scale as $\left(R^{d}-R_{0}{ }^{d}\right) \sim c_{\mathrm{P}}$.

Fig. 6 shows $R_{\mathrm{H}}{ }^{d}$, where $R_{\mathrm{H}}$ is the hydrodynamic radius of Cel-g-PS-I aggregates at $c_{\mathrm{SDS}}=100 \mathrm{mM}$ and $d=2.53$ is their fractal dimension obtained from SAXS, as a function of $c_{\mathrm{P}}$ (curve 1). In accordance with the above-mentioned theoretical considerations, the dependence is linear, providing the extrapolated value of the initial hydrodynamic radius, $R_{\mathrm{H}, 0}$ $=122 \mathrm{~nm}$. The mean radius of the Cel-g-PS macromolecules on the micrographs of the aqueous dispersions (Fig. 5a-d) is lower (about $50 \mathrm{~nm}$ ) but it is necessary to keep in mind that we compare the number averaged value with the hydrodynamic radii from DLS which is based on the z-averaged diffusion coefficient. 


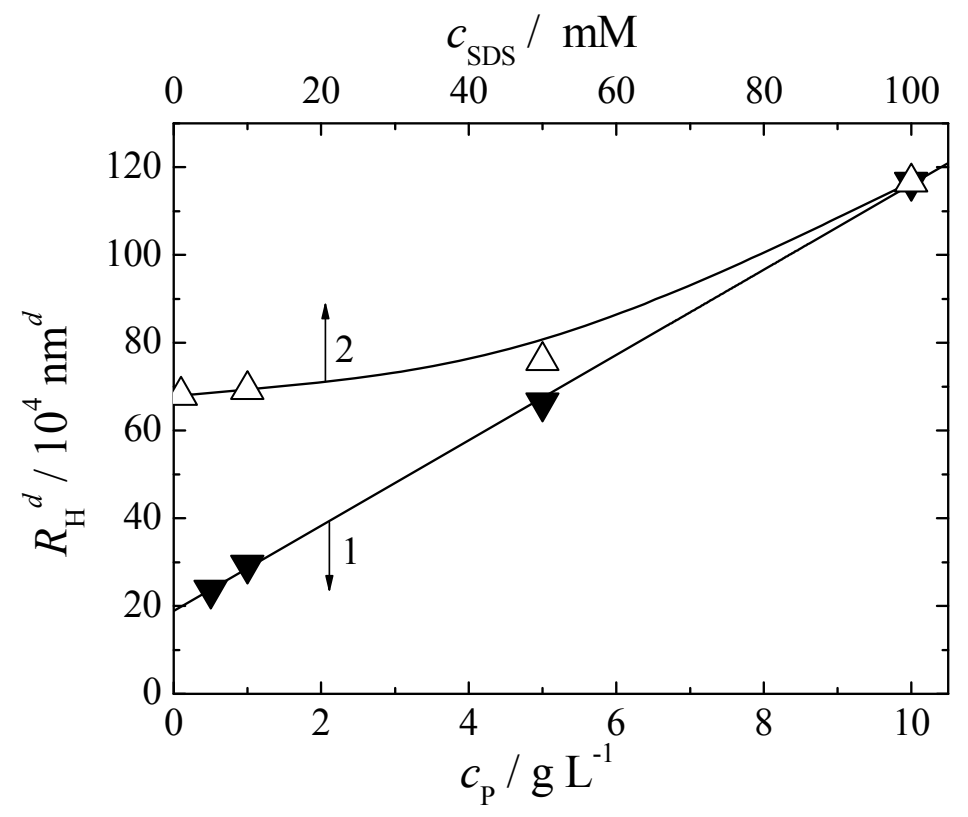

Fig. 6. $R_{\mathrm{H}}{ }^{d}$, where $R_{\mathrm{H}}$ are the hydrodynamic radii of the nanoprecipitated particles of Cel-g-PS-I and $d=2.53$ is their fractal dimension, as functions of the copolymer concentration in the 1,4-dioxane solution, $c_{\mathrm{P}}($ curve 1$)$, and of SDS concentration in the aqueous solution, $c_{\mathrm{SDS}}$ (curve 2).

In the case that we follow the $R_{\mathrm{H}}$ of the aggregates as a function of $c_{\mathrm{SDS}}$ (at $c_{\mathrm{P}}=1$ $\mathrm{mg} / \mathrm{mL}$ ), the slight decrease in the hydrodynamic radius with the decreasing SDS concentration is observed (Fig. 6, curve 2). (SDS concentrations below $1 \mathrm{mM}$ were insufficient for the stabilization of the nanoparticles and macroscopic precipitation of the copolymer was observed.) This behavior can be explained by the decrease in the screening of electrostatic repulsion between individual Cel-g-PS-I macromolecules with adsorbed SDS which dominates over of the effect of the increased rate of the SDS adsorption at higher SDS concentrations.

\section{Conclusions}

In this paper, we have studied the structure of nanoparticles in aqueous dispersions prepared by nanoprecipitation of high-molar-mass densely grafted cellulose-graft-polystyrene copolymers from 1,4-dioxane solutions. Scattering and transmission electron microscopy measurements show that the aggregation of Cel-g-PS in the presence of sodium dodecyl sulfate leads to the formation of loose clusters of individual collapsed macromolecules, the 
size of which is kinetically controlled and can be tuned by changing the Cel-g-PS concentration in the 1,4-dioxane solution which is mixed with the aqueous SDS. The aggregates are electrostatically stabilized by entrapped SDS anions (SAXS in the high- $q$ region indicates that the particles contain densely packed SDS micelles) which cannot escape from the interior of the particles and keep them negatively charged when the dispersion is subject to dialysis and excess surfactant molecules are removed from the solution. Therefore we can conclude that the aggregation of large branched macromolecules resembles rather that of solid colloidal particles than that of small polymers because the individual collapsed macromolecules in the aggregate do not fully penetrated and remain only loosely connected.

\section{Associated Content}

Supporting Information file containing STEM images of (i) Cel-g-PS particles from freezedried 1,4-dioxane solutions and (ii) hydrated Cel-g-PS particles from aqueous dispersions obtained in the environmental mode (wet-STEM).

\section{Acknowledgments}

The financial support from the Czech Science Foundation (Grant P106-12-0143) and from the Technology Agency of the Czech Republic (Grant TE01020118) is gratefully acknowledged. The kind support from Clement Blanchet at the P12 BioSAXS beamline (EMBL/DESY, PETRA III)

\section{References}

(1) Taylor, D.J.F.; Thomas, R.K.; Penfold, J. Polymer/surfactant interactions at the air/water interface. Adv. Colloid Interface Sci. 2007, 132, 69-110.

(2) Tam, K.C.; Wyn-Jones, E. Insights on polymer surfactant complex structures during the binding of surfactants to polymers as measured by equilibrium and structural techniques. Chem. Soc. Revs. 2006, 35, 693-709.

(3) Langevin, D. Complexation of oppositely charged polyelectrolytes and surfactants in 
aqueous solutions. A review. Adv. Colloid Interface Sci. 2009, 147-148, 170-177.

(4) Zhou, S.; Chu, B. Assembled materials: Polyelectrolyte-surfactant complexes. Adv. Mater. 2000, 12, 545-556.

(5) Vitorazi, L.; Berret, J.F.; Loh, W. Self-Assembly of Complex Salts of Cationic Surfactants and Anionic-Neutral Block Copolymers. Dispersions with Liquid-Crystalline Internal Structure. Langmuir 2013, 29, 14024-14033.

(6) Uchman, M.; Štěpánek, M.; Prévost, S.; Angelov, B.; Bednár, J.; Appavou, M.S.; Gradzielski, M.; Procházka, K. Coassembly of Poly(ethylene oxide)-blockpoly(methacrylic acid) and N-Dodecylpyridinium Chloride in Aqueous Solutions Leading to Ordered Micellar Assemblies within Copolymer Aggregates. Macromolecules 2012, 45, 6471-6480.

(7) Antonietti, M.; Landfester, K. Polyreactions in miniemulsions.

Prog. Polym. Sci. 2002, 27, 689-757.

(8) Bourgeat-Lami, E.; Farzi, G.A.; David, L.; Putaux, J.L.; McKenna, T.F.L. Silica Encapsulation by Miniemulsion Polymerization: Distribution and Localization of the Silica Particles in Droplets and Latex Particles. Langmuir 2012, 28, 6021-6031.

(9) Hecht, L.L.; Wagner, C.; Landfester, K.; Schuchmann, H.P. Surfactant Concentration Regime in Miniemulsion Polymerization for the Formation of MMA Nanodroplets by High-Pressure Homogenization. Langmuir 2011, 27, 2279-2285.

(10) Mora-Huertas, C.E.; Fessi, H.; Elaissari, A. Polymer-based nanocapsules for drug delivery. Int. J. Pharm. 2010, 385, 113-142.

(11) D'Addio, S.M.; Prud'homme, R.K. Controlling drug nanoparticle formation by rapid precipitation. Adv. Drug Delivery Revs. 2011, 63, 417-426.

(12) Xie, H.; She, Z.G.; Wang, S.; Sharma, G.; Smith, J.W. One-Step Fabrication of Polymeric Janus Nanoparticles for Drug Delivery. Langmuir 2012, 28, 4459-4463.

(13) Jager, A.; Gromadzki, D.; Jager, E.; Giacomelli, F.C.; Kozlowska, A.; Kobera, L.; Brus, J.; Ř́íhová, B.; El Fray, M.; Ulbrich, K.; Štěpánek, P. Novel "soft" biodegradable nanoparticles prepared from aliphatic based monomers as a potential drug delivery system. Soft Matter 2012, 8, 4343-4354.

(14) Stepanyan, R.; Lebouille, J.G.J.L.; Slot, J.J.M.; Tuinier, R.; Stuart, M.A.C. Controlled Nanoparticle Formation by Diffusion Limited Coalescence. Phys. Rev. Lett. 2012, 109, Art. No. 138301.

(15) Lin, M.Y.; Lindsay, H.M; Weitz, D.A.; Ball, R.C.; Klein, R.; Meakin, P. 
Universality in colloid aggregation. Nature 1989, 339, 360-362.

(16) Raus, V.; Štěpánek, M.; Uchman, M.; Šlouf, M.; Látalová, P.; Čadová, P.; Netopilík, M.; Kříž, J.; Dybal, J.; Vlček, P. Cellulose-Based Graft Copolymers with Controlled Architecture Prepared in a Homogeneous Phase. J. Polym. Sci. A Polym. Chem. 2011, 49, 4353-4367.

(17) Dušek, K.; Netopilík, M.; Kratochvíl, P. Nonuniformities of Distributions of Molecular Weights of Grafted Polymers. Macromolecules 2012, 45, 3240-3246.

(18) Franke, D.; Kikhney, A.G.; Svergun, D.I. Automated acquisition and analysis of small angle X-ray scattering data. Nucl. Instrum. Methods A 2012, 689, 52-59.

(19) http://www.embl-hamburg.de/biosaxs/manuals/primus-qw.html

(20) Haw, M.D.; Poon, W.C.K.; Pusey, P.N. Structure and arrangement of clusters in cluster aggregation. Phys. Rev. E 1997, 56, 1918-1933.

(21) Bantle, S.; Schmidt, M.; Burchard, W. Simultaneous Static and Dynamic Light Scattering. Macromolecules 1982, 15, 1604.

(22) Lei, N.; Safinya, C. R.; Roux, D.; Liang, K. S. Synchrotron x-ray-scattering studies on the sodium dodecyl sulfate-water-pentanol-dodecane L3 sponge phase. Phys. Rev. E 1997, 56, 608-613.

(23) Hajduová, J.; Procházka, K.; Šlouf, M.; Angelov, B.; Mountrichas, G.; Pispas, S.;

Štěpánek, M. Polyelectrolyte-Surfactant Complexes of Poly[3,5-

bis(dimethylaminomethyl)-4-hydroxystyrene]-block-poly(ethylene

oxide) and Sodium Dodecyl Sulfate: Anomalous Self-Assembly

Behavior. Langmuir 2013, 29, 5443-5449. 